CASE REPORT

\title{
Microscopic EXTRAOVARIAN SEX CORD PROLIFERATION: REPORT OF A CASE WITH BILATERAL FALLOPIAN TUBE INVOLVEMENT AND A COMPREHENSIVE MOLECULAR ANALYSIS
}

\author{
Pavel Dundr, Zuzana Věcková, Ivana Tichá, Jan Hojný, Kristýna Němejcová, \\ Michaela BÁRTŮ
}

Institute of Pathology, First Faculty of Medicine, Charles University and General University Hospital in Prague, Czech Republic

\begin{abstract}
We report a case of a 58-year-old female with microscopic extraovarian sex cord proliferations affecting both Fallopian tubes. Molecular analysis showed likely pathogenic germline missense mutations of the KDM5A and KMT2D genes. However, mutations of other genes, including FOXL2 and STK11, were not detected. Our case represents the $12^{\text {th }}$ case of extraovarian sex cord proliferation reported in the literature to date. This is the first time that a molecular genetic analysis of the lesion has been performed, and it showed a wild-type FOXL2 gene, which represents another argument supporting the estimated benign nature of these rare lesions.
\end{abstract}

Key words: extraovarian sex cord stromal proliferation, Fallopian tube, FOXL2.

\section{Introduction}

Heterotopic extraovarian sex cord stromal proliferations are rare lesions which have only recently been described in the literature $[1,2,3]$. These proliferations can be found in several areas including the Fallopian tubes, paraovarian connective tissues, the pelvic side wall, and the serosa of the appendix. The histological spectrum of these proliferations is broad and encompasses lesions resembling an adult granulosa cell tumor (AGCT), sex cord tumor with annular tubules (SCTAT), ovarian fibroma, ovarian cortical stroma, adenofibroma, and cystadenofibroma. There is also a recent report, which described four cases of ovarian hilar proliferations resembling the structures of Sertoli cell tumors [4]. Given the more extensive examination of adnexal tissues, especially in patients undergoing prophylactic salpingo-oophorectomy, it is to be expected that these proliferations will probably be detected with increased frequency, and pathologists should be aware of these lesions. We report a case of heterotopic mi- croscopic sex cord proliferation affecting both Fallopian tubes, resembling AGCT and SCTAT, which represents only the 12 th case described in the literature to date. This is the first time that a comprehensive molecular analysis of this rare lesion has been performed.

\section{Clinical history}

The patient was a 58-year-old female with a history of a high grade squamous intraepithelial lesion (HSIL) and adenocarcinoma in situ of the uterine cervix. She was treated by cervical conization (July 2018), which showed positive surgical margins. A re-conization was performed two months later and showed only a low grade squamous intraepithelial lesion (LSIL) with free surgical margins. Eventually, the patient underwent a hysterectomy with bilateral salpingectomy in January 2019. Clinically, the ovaries were normal in size, and a subsequent ultrasound examination showed no ovarian or pelvic masses. The eight months of follow-up were uneventful. 


\section{Material and methods}

\section{Immunohistochemistry}

Selected sections from both Fallopian tube lesions were analyzed immunohistochemically using the avidin-biotin complex method with antibodies directed against the following antigens: inhibin (clone R1, ready-to-use, Dako, Glostrup, Denmark), WT-1 (clone 6F-H2, 1:100, Dako), calretinin (clone DAKCalret 1, 1:200, Dako), estrogen receptor (clone SP1, 1:100, Thermo Fischer Scientific, Waltham, MA, USA), progesterone receptor (clone 15, 1:100, Novocastra, Leica Biosystems, IL, USA), S100-A1 protein (1:600, Dako), CD56 (clone 1B6, 1:50, Novocastra), smooth muscle actin (clone 1A4, 1:400, SigmaAldrich, MS, USA), and PAX8 (1:50, Cell Marque, CA, USA).

\section{Molecular analysis}

DNA was isolated from formalin-fixed, paraffin-embedded (FFPE) tissue by the cobas ${ }^{\circledR}$ DNA Sample Preparation kit (Roche; Germany). Macrodissection was performed in order to enrich the content of lesional cells. Tissue from both Fallopian tube lesions and adjacent non-lesional normal tissue was used for sequence capture next-generation sequencing (NGS) which was performed with $200 \mathrm{ng}$ of gDNA ( $70 \%$ of lesional cells). The sample libraries were constructed using KAPA HyperPlus kit (Roche) according to the manufacturer's protocol. Libraries were enriched using custom-designed hybridization probes (Nimblegen, Roche; target area 1020 kbp; 271 genes) according to the SeqCap EZ Library protocol (Roche). The samples were pair-end sequenced using NextSeq 500/550 Mid Output Kit v2.5 (150 Cycles; Illumina) on NextSeq 500 instrument (Illumina). Following the raw sequencing, the data was demultiplexed, PCR-duplicate reads and low-quality reads were removed, and the resulting reads were aligned to the human genome (GRCh37/hg 19) with an average coverage of $726 \times-948 \times$ in the target area. Only the nonsynonymous variants in exons and adjacent intronic regions with minimal coverage $100 \times$ and frequency $>5 \%$ were evaluated, and manually controlled using an Integrative Genomic Viewer (Broad Institute). Due to the generally lower quality of the FFPE samples, copy number variations were not evaluated.

The evaluation of the functional or clinical importance of the detected missense variants was carried out with the widely used in silico prediction tools (part of the NextGENe software: dbNSFP v 2.9 - set of algorithms for predicting the impact of the variant on protein function, according to the sequence conservation and population frequency; ClinVar; dbscSNV for evaluating the impact of splice variants). The impact of the prioritized variants was also assessed using 14 different in silico prediction tools implemented in the Variant Effect Predictor (VEP, Ensembl, http:// grch37.ensembl.org/Homo_sapiens/Tools/VEP/). The rank score, which ranged from 0 (= likely benign) to 1 (= likely pathogenic), was evaluated in all the selected predictors. We considered a variant as "likely pathogenic" if at least $7 / 14$ of the selected predictors scored the variant as $\geq 0.8$.

\section{Ethics statement}

The project has been approved by the Ethics Committee of General University Hospital in Prague in compliance with the Helsinki Declaration.

\section{Results}

Grossly, the uterine cervix was crater-shaped due to the previously performed procedures. There was a small, subserosal uterine leiomyoma measuring $15 \mathrm{~mm}$ in diameter. Otherwise, macroscopically the hysterectomy specimen was without any apparent changes. The Fallopian tubes measured $55 \mathrm{~mm}$ (left) and $60 \mathrm{~mm}$ (right), with a diameter of up to $4 \mathrm{~mm}$. Both Fallopian tubes were grossly without apparent lesions.

The entire uterine cervix was processed, and microscopically it was without any residual squamous intraepithelial lesion. The randomly sampled endometrium was inactive and the subserosal leiomyoma was of a usual appearance. The Fallopian tubes were processed according to our institutional guidelines applicable for benign salpingectomy specimes. In these cases we use a modified SEE-FIM protocol, in which the fimbrial part of the Fallopian tubes is processed in longitudinal sections in toto, while the remaining part of each tube is examined in three transversal sections. Microscopically, there were small sex cord stromal proliferations found in the fimbrial parts of both Fallopian tubes. The largest dimension of these proliferations (as measured on the slide) was $3.2 \times 0.7 \mathrm{~mm}$ in the left tube, and $2.4 \times 1.4 \mathrm{~mm}$ in the right tube. The proliferations consisted of well demarcated nests of cells with cribriform appearance, with spaces containing eosinophilic material, resembling SCTAT. There were also areas which resembled solid AGCT with regressive changes, and some microfollicles and structures resembling Call-Exner bodies (Figs. 1, 2). The appearance of the lesional cells was regular, with occasional nuclear grooves, and without any apparent mitoses. In these areas, small foci of hyalinization were present.

Immunohistochemically, the lesional cells were strongly and diffusely positive for inhibin, calretinin, and WT-1 (Figs. 3, 4). Estrogen and progesterone receptors were only focally, weakly positive. PAX8, 


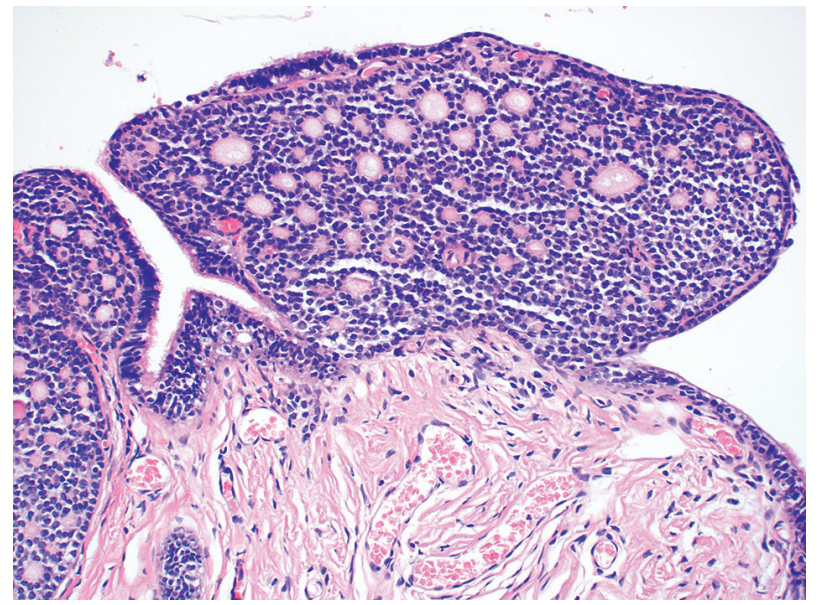

Fig. 1. A nest of regular sex cord cells, resembling sex cord tumor with annular tubules $(200 \times$, hematoxylin and eosin)

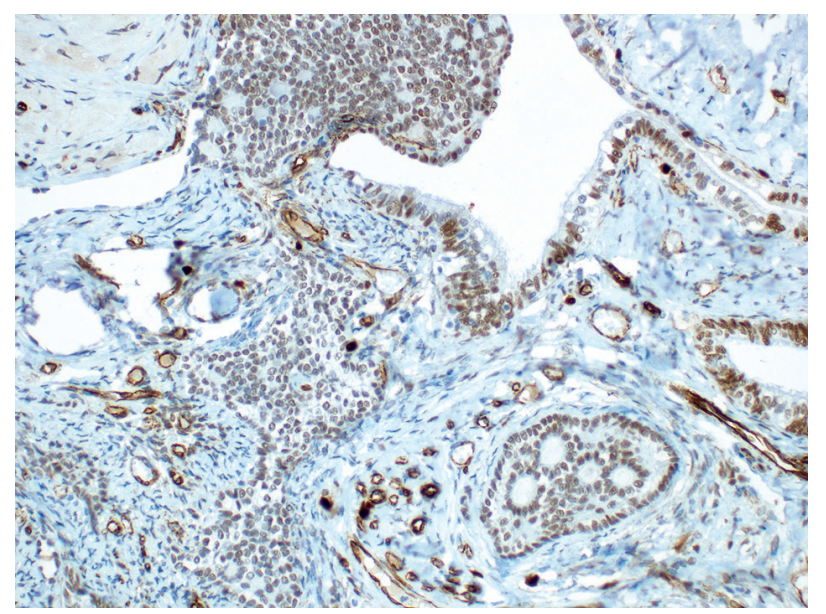

Fig. 3. Positivity of lesional cells for WT-1 $(200 \times)$

CD56, S-100 protein and smooth muscle actin were negative.

Molecular analysis was fully performed on 227/271 genes with sufficient coverage $(>100 \times)$ in all the 3 tested samples (two independent samples of the lesions and one sample of a normal tissue). The analysis revealed a non-synonymous germline heterozygous variant NM 001042603.1:c.4831G > A; NP 001036068:p.(A1611T) in the KDM5A gene (also known as JARID1A or RBBP2). This variant is not reported in ClinVar or Cosmic, however, it is recorded in the dbSNP database under the name rs200109736. This variant was deemed as likely pathogenic by the in silico analysis. Another result of the analysis was the identification of a heterozygous germline variant NM_003482.3:c.941C > G, NP 003473.3:p.(S314C), rs1043654062, which was found in the KMT2D gene (also known as MLL2). This variant is considered as VUS by ClinVar, and was deemed as likely pathogenic by the in silico analysis. Additionally, there was a homozygous ger-

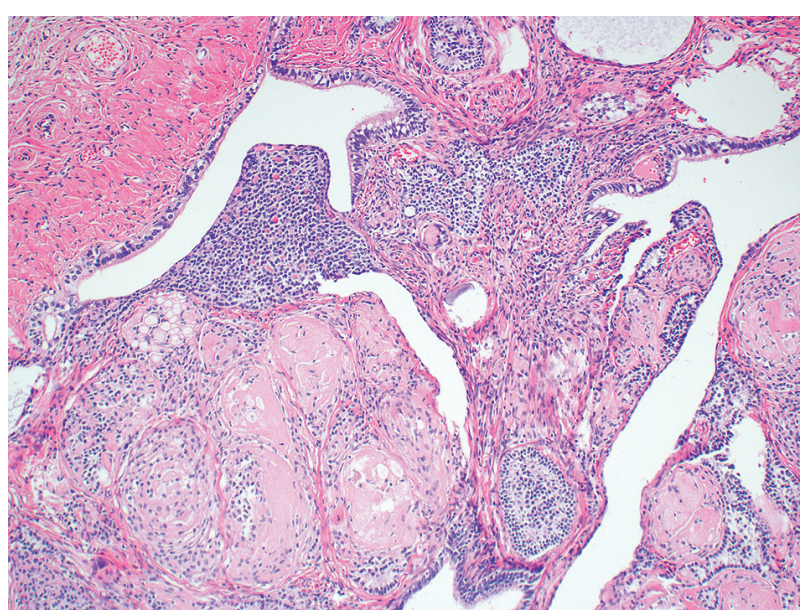

Fig. 2. Multiple nests of sex cord cells with some regressive changes with hyalinizations $(100 \times$, hematoxylin and eosin)

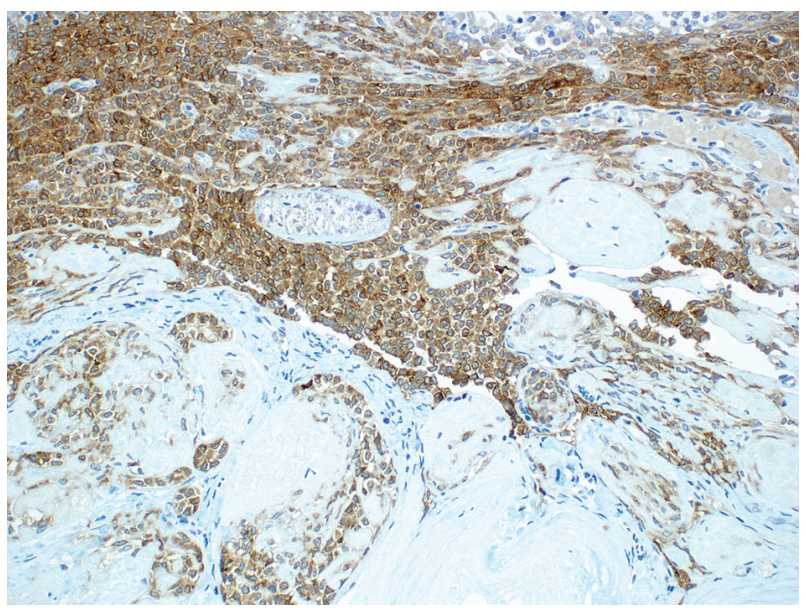

Fig. 4. Positivity of lesional cells for inhibin $(200 \times)$

mline polymorphism detected in the FGFR 4 gene, NM_213647.2: c.1162G>A， NP_998812.1:p. (G388R), rs351855 (considered as VUS by ClinVar; benign by the in silico analysis); and a heterozygous germline polymorphism was also found in the NBN gene, NM_002485.4:c.511A>G, NP_002476.2:p. (I171V), rs61754966 (considered as VUS by ClinVar; benign by the in silico analysis). No pathogenic mutations were found in either the FOXL2 or STK11 gene, which are commonly mutated in AGCT or patients with Peutz-Jeghers syndrome.

\section{Discussion}

The spectrum of extraovarian sex cord proliferations is broad and, from a practical point of view, the finding bears several consequences, especially for differential diagnostics. Heterotopic hilar cell proliferation is a rare finding, but its diagnosis is usually straightforward [5]. However, recently a range of extraovarian proliferations of other structures of sex 
Table I. Clinico-pathological features of extraovarian sex cord proliferations

\begin{tabular}{|c|c|c|c|c|c|}
\hline REFERENCE NO. & Case no. & AGE (YEARS) & SIZE & LOCATION & FOLLOW-UP \\
\hline Our case & 1 & 58 & $3.2 \times 0.7 \mathrm{~mm} / 2.4 \times 1.4 \mathrm{~mm}$ & Both FT & 8 months \\
\hline \multirow[t]{6}{*}{$(1)$} & 2 & 58 & $<1 \mathrm{~mm}$ & FT fimbriae & 48 months \\
\hline & 3 & 39 & $<1 \mathrm{~mm}$ & FT fimbriae & NA (recent case) \\
\hline & 4 & 48 & $<1 \mathrm{~mm}$ & Appendiceal serosa & NA (recent case) \\
\hline & 5 & 23 & $<1 \mathrm{~mm}$ & Right pelvic side wall & 11 months \\
\hline & 6 & 58 & $<1 \mathrm{~mm}$ & $\begin{array}{c}\text { Para-ovarian tissues } \\
\text { and ovarian capsular } \\
\text { adhesions }\end{array}$ & 2 years \\
\hline & 7 & 39 & $3,75 \mathrm{~mm}$ & FT isthmus & 6 years \\
\hline$(2)$ & 8 & 44 & $1 \mathrm{~mm}$ & FT bellow fimbriae & 2 years \\
\hline \multirow[t]{4}{*}{ (3) } & 9 & 25 & NA & Paratubal cyst & NA \\
\hline & 10 & 70 & NA & Paratubal tissues & NA \\
\hline & 11 & 28 & NA & FT fimbriae & NA \\
\hline & 12 & 27 & NA & Paratubal cyst & NA \\
\hline
\end{tabular}

FT - Fallopian tube; NA - not available

cord stromal proliferations have been described, and reported to resemble AGCT, SCTAT, Sertoli cells tumor, ovarian stromal tissue or ovarian fibroma, and lesions of Müllerian differentiation resembling adenofibroma and cystadenofibroma (serous and Brenner type). The differential diagnosis of these lesions may clearly be more complicated $[1,2,3]$. Generally, the histogenetic origin of these lesions is regarded to be heterotopic, representing non-neoplastic proliferations of embryonic remnants [1]. However, other possible mechanisms cannot be excluded with certainty, including implantation of tissue fragments from the ovary during ovulation, microscopic neoplasia, or theoretically also metaplasia [3]. In addition, we believe that in the cases resembling SCTAT the possibility of Peutz-Jeghers syndrome should be considered, unless it is shown with certainty that the finding is unrelated. This hypothesis, however, requires the analysis of more cases.

Concerning the AGCT and SCTAT-like proliferations, despite the rarity of these lesions (as only 11 cases have been reported to date), pathologists should be aware of the existence of these changes in extraovarian localizations. If this proliferation is found in a biopsy, the differential diagnosis should primarily include the possibility of a dissemination of a neoplastic process of another primary origin, either ovarian or extraovarian. Sex cord stromal tumors, including AGCT and SCTAT, may in fact also primarily arise in extraovarian locations including the Fallopian tube, broad ligament, retroperitoneum, adrenal gland and umbilical hernia sac $[6,7,8]$. In this context a thorough examination of the patient is necessary, especially in cases where the lesion is an incidental finding in a salpingectomy specimen without an oophorectomy. However, even after the elimination of another possible primary source, the biological nature of these processes is still uncertain - all the cases reported so far have been described as benign, but the follow-up is often short and the pathologist's information for the clinicians should expressly state that although the nature of the process is probably benign, a close follow-up is appropriate. The benignity of the process, besides the small size of the so far reported lesions (which is usually up to $3.75 \mathrm{~mm}$ ), is also supported by the occurrence of bilateral tubal involvement, as was present in our case (after excluding dissemination from another primary source as we believe that the occurrence of a simultaneous bilateral minute AGCT/SCTAT is highly improbable). The clinico-pathological features of all reported cases are summarized in Table I.

Another aspect supporting the benignity of the lesion may be found in the molecular testing focusing on the FOXL2 gene. The molecular analysis can be of interest, as more than $90 \%$ of AGCT is characterized by a somatic missense FOXL2 mutation NM_023067.4:c.402C>G, NP_NP_075555.1:p. $(\mathrm{C} 134 \mathrm{~W})$, rs 1057519865 in the codon 134 [9, 10]. However, to date no such analysis of extraovarian sex cord proliferations has been successfully performed, which is probably partly related to the small size of the lesions. We have found only one mention of an attempted testing, which showed inconclusive results due to the low DNA concentration [1]. In our case, the molecular analysis of both Fallopian tube lesions and non-lesional normal tissue was successful. We used our in-house mid-sized NGS panel consisting of 271 genes $(1020 \mathrm{kbp})$. We detected a germline likely pathogenic (in silico prediction) mutation 
p.(A1611T) in the KDM5A gene. This gene is located in the Zinc-finger PHD3 functional domain, which spans the amino acids 1607-1661 and is involved in the recognition of the $\mathrm{H} 3 \mathrm{~K} 4$ methylation status [11]. The KDM5A protein is a lysine demethylase $5 \mathrm{~A}$, which specifically demethylates the lysine 4 of histone $\mathrm{H} 3$. It has been reported that a genetic deletion of KDM $5 A$ resulted in upregulation of $\mathrm{p} 53$ in multiple cancer cell lineages and inhibits tumor growth in a p53-dependent manner [12]. The other detected likely pathogenic (in silico prediction) KMT2D variant p.(S314C) is located in the Zinc-finger RING-type 2 domain, which spans the amino acids 276-321. The protein product of this gene is a lysine methyltransferase 2D, which methylates the lysine 4 position of histone $\mathrm{H} 3$, which is an interestingly contradictory effect compared to KDM5A's function.

The analysis also detected other germline variants of the FGFR 4 and NBN gene, which were classified as of unknown significance (VUS) and predicted benign by in silico analyses. The FOXL2 gene, as well as the STK11 gene, were revealed to be wild type, which goes against the possibility of a tumorous origin of the lesions (FOXL2), and also against the association with Peutz-Jeghers syndrome (STK11).

Other possible differential diagnosis includes metastases of other tumors (such as small cell carcinoma or carcinoid), benign conditions (such as Walthard nests or mesothelial proliferations), and displaced granulosa cells from the ovary. Concerning the possible metastatic spread of neuroendocrine tumors, this suspicion may arise especially in cases with crushing artifacts. However, in these cases immunohistochemistry would be helpful, confirming either a sex cord stromal lineage or neuroendocrine lineage. Walthard nests and mesothelial proliferations are rather a theoretical possibility, once again easily solved by the immunohistochemical profile of the lesions. Excluding displaced granulosa cells from the differential diagnosis may be more difficult, as these would have the same immunohistochemical profile. However, the displaced granulosa cells are characterized by sheets of cells commonly showing crushing artifacts, without any specific morphological pattern characteristic of extraovarian sex cord stromal proliferations. Moreover, they are commonly located inside the vascular channels $[13,14,15]$.

In conclusion, we described an additional case of extraovarian sex cord proliferation resembling AGCT and SCTAT. Despite the benign behavior of all lesions described to date, the benign nature of these lesions cannot yet be unequivocally confirmed. Given this, the lesions should be viewed with caution and a thorough examination of the patients with subsequent close follow-up should be recommended. This is the first time that a comprehensive molec- ular analysis of the lesion has been performed, and the results have excluded the mutation of the FOXL2 gene occurring in a vast majority of AGCT. This finding represents another piece of evidence pointing to the benign character of these proliferations, however, an analysis of more cases is needed. The analysis of the STK11 gene performed in our case also excluded the possible association with Peutz-Jeghers syndrome.

This work was supported by Ministry of Health, Czech Republic (Conceptual development of research organization 64165, General University Hospital in Prague), by Charles University (Project Progres Q28/LF1, and UNCE 204065), by European Regional Development Fund, project EF16_013/0001674 and BBMRI_ CZ LM2018125, and by OPPK (Research Laboratory of Tumor Diseases, CZ.2.16/3.1.00/24509). The authors wish to thank Zachary H.K. Kendall, B.A. for the English editing.

The authors declare no conflict of interest.

\section{References}

1. McCluggage WG, Stewart CJ, Iacobelli J, et al. Microscopic extraovarian sex cord proliferations: an undescribed phenomenon. Histopathology 2015; 66: 555-564.

2. Pennacchia I, Arena V, Federico F, et al. Microscopic Extraovarian Sex Cord Proliferations: Report of the Seventh Case With 2 Year Follow-up. Int J Gynecol Pathol 2018; 37: 347-348.

3. Longworth A, Ganesan R, Yoong AKH, et al. Microscopic Heterotopic Extraovarian Sex Cord-Stromal Proliferations: Expanding the Histologic Spectrum. Int J Gynecol Pathol 2018; 37: 35-43.

4. Taylor J, Clarke BA, Hasan NU, et al. Ovarian hilar proliferations resembling Sertoli cell tumours: microscopic neoplasms or non-neoplastic remnants? Histopathology 2016; 68: 596602 .

5. Hirschowitz L, Salmons N, Ganesan R. Ovarian hilus cell heterotopia. Int J Gynecol Pathol 2011; 30: 46-52.

6. Keitoku M, Konishi I, Nanbu K, et al. Extraovarian sex cord-stromal tumor: case report and review of the literature. Int J Gynecol Pathol 1997; 16: 180-185.

7. Omori M, Kondo T, Fukushima J, et al. Extraovarian Fibroma With Minor Sex Cord Elements: A Case Report and Literature Review. Int J Surg Pathol 2017; 25: 472-476.

8. Shah R, McCluggage WG. Unclassifiable Malignant Extraovarian Sex Cord-Stromal Tumors: Report of 3 Cases and Review of Extraovarian Sex Cord-Stromal Tumors. Int J Gynecol Pathol 2017; 36: 438-446.

9. Kommoss S, Anglesio MS, Mackenzie R, et al. FOXL2 molecular testing in ovarian neoplasms: diagnostic approach and procedural guidelines. Mod Pathol 2013; 26: 860-867.

10. Shah SP, Kobel M, Senz J, et al. Mutation of FOXL2 in granulosa-cell tumors of the ovary. N Engl J Med 2009; 360: 27192729.

11. Wang GG, Song J, Wang Z, et al. Haematopoietic malignancies caused by dysregulation of a chromatin-binding PHD finger. Nature 2009; 459: 847-851.

12. Hu D, Jablonowski C, Cheng PH, et al. KDM5A Regulates a Translational Program that Controls p53 Protein Expression. iScience 2018; 9: 84-100. 
13. Duncan DL, Rambally BS, Lininger RA, et al. Displaced granulosa cells in the fallopian tube mistaken for metastatic granulosa cell tumor. Int J Gynecol Pathol 2013; 32: 35-37.

14. Chui MH, Chapman WB, Clarke BA. Displaced granulosa cells within the ovarian stroma in a BRCA1 mutation carrier. Int J Gynecol Pathol 2014; 33: 423-424.

15. Vydianath B, Ganesan R, McCluggage WG. Displaced granulosa cells in the fallopian tube mimicking small cell carcinoma. J Clin Pathol 2008; 61: 1323-1325.

\section{Address for correspondence}

\section{Pavel Dundr}

Institute of Pathology

First Faculty of Medicine

Charles University and General University Hospital in Prague

Prague, Czech Republic

e-mail: pavel.dundr@vfn.cz 